\title{
Is external control important for internal control?
}

\author{
Evo Busseniers
}

Global Brain Institute, VUB, Belgium

In cybernetics, the basic assumption is that systems aim for control. But what and how are they controlling? I differentiate two categories of control: internal and external control. In external control, one starts from outside, and tries to determine the environment completely. While with internal control, one's own aspirations are taken as a starting point, and useful synergies with the environment are sought. I'll define these concepts in cybernetic terms, and argue why they are not necessary related. There are two aspects in this difference. The first lies in the locality: is there an aim for global control, or only for control in the immediate environment? There is also a difference in what one tries to change. An agent could adapt its links, or try to change either the methods or the goals of its neighbors. Several of these cases will be further explored by giving an existing model that falls under this circumstances. All of these models will be put into an overarching framework. The model of controllability is a model about global control, while the others model local control, based on neural networks or perceptual control theory.

url:

\section{Introduction}

A growing number of people feel like they don't have control over their own life, that the path they should follow is already predetermined. They have the impression somebody or something is controlling them, and they would like to have control over their own life. But often it is presented as if you only have two choices: either dominate, or being dominated. In speaking about control, there is no difference made between being in control over your own life, and controlling others. This is why I introduce the concepts of internal and external control. If one attempts to control its own situation, to fulfill its aspirations, I speak of internal control. External control means one attempts to determine environmental behavior. The goal is to control everything completely.

External control can be used in order to get internal control. But I argue that this is not the only way: it is possible to change something without controlling others. One can find synergies in her environment, so that he can develop to the fullest without standing in the way of other people's development. Thus, there are different strategies to get internal control.

If one uses external control, one keeps things out of their natural state, the equilibrium state, for its own profit. People are pushed in a state where someone wants them to be, which is unnatural for them, and which they often don't want. This requires constant energy to keep this state.

The second method is that one considers that people can take different paths in life, that there are different equilibria. How their life develops depends on what they encounter and whether they are

Corresponding author: Evo Busseniers; e-mail: ebusseni@vub.ac.be 
empowered. Thus, there are different bifurcation points in one's life, where going one way isn't always clearly better than the other. People can then align their paths so that one's path is beneficial for the other. One just interacts with others so that they have the possibility to take the other path and are empowered to do so. An example is the distribution of leaflets with information about a certain oppressive practice, and ways one can resist to it. People have the choice to ignore it, but at least they now know about it, and are empowered to do something against it. This is something completely different than telling people what they should do.

An example of the different strategies for control, is the difference between traditional agriculture and permaculture. In traditional agriculture, the farmer tries to control the land completely: he removes all the organisms that don't give him food directly, often just keeping one crop which he tries to optimize to get as much as possible out of it. This requires constant energy: he'll need fertilizer because the soil will get depleted from having only one crop that takes all its nutrients, and he'll need some products and machines to keep the weeds and insects away. In permaculture, the whole ecosystem is kept. One tries to interfere as little as possible in all constituent sub-ecosystems. But different kinds of ecosystems are possible, depending on small differences. The idea is that you watch and learn from how nature works, and build an ecosystem where you also get out what you want. The system will maintain itself, thus it could in theory be sustained indefinitely, in a permanent agriculture, hence the name.

Another easier and more fictive example is the different ways you can deal with rain. You could just accept it, "It's raining, I'm getting wet, and I can do nothing about it". Or you could try to influence the weather. But you can also build a shelter. In this way, you gain control over your life without having to control the rain.

A related concept is 'Risk board mentality': the belief that contact between people who are different must result in a missionary relationship, with one converting the other (Gelderloos, 2007).

Gelderloos argues that there can be a mutual influence, it's not either dominate or being dominated. You don't have to conquer the world to get control over your life.

This work can also be related to the idea of antifragility (Taleb, 2012). A system is called antifragile if it gets stronger after a shock. The principle is that one makes the system more antifragile, but one doesn't predetermine how it should behave exactly. With a strict plan, a blueprint, of how everything should be, the system will be pretty fragile: as soon as something is a little bit different than planned, everything falls apart. This is an argument for why aiming for external control could make a system more fragile.

The concept of two categories of constraints applied by management processes (Stewart, 2014) is similar to these different strategies for control. Prescriptive constraints specify more or less precisely the particular outcomes that occur in the managed group. Evolvability resides in the manager, since the other entities mostly just do as they are told. With enabling constraints, the interests of group members get aligned with the interests of the group as a whole. Then, when an agent acts in its own self-interest, it is also in the interest of the group. The advantage of this type is that it uses the local knowledge and the diversity of the group.

Stewart also cites Salthe (Salthe, 2013) who states that constraints can arise in two ways: upper-level constraints arise external to the dynamic of entities, while lower-level constraints are fixed, internal features of the interacting entities that can influence how entities behave. Both influence the dynamic, but they aren't influenced in return, which is Stewart's definition of power. 
In the previous I always somehow assumed there was one agent wanting control. It is important to keep in mind that being controlled doesn't necessarily have to happen by one agent. In "Perceptual control and social power"(McClelland, 1994), McClelland argues that social power is alignment. It is when lots of people align to the same goal, that it is difficult to do something different. Power thus doesn't reside in one individual.

Following, I will first explore how we can define internal and external control in cybernetic terms. Then I will put several models of control in a general framework, which is based on the model of Mesarovic (Mesarovic, Macko, \& Takahara, 200o). The idea is that there are two aspects in the difference between internal and external control. First, there is a difference between acting locally or globally. The other difference lies in what one tries to change: either the links, methods or goals of other agents. The model of controllability implies an aiming for global control by adapting goals. The other models work with local control. In the model of self-organized control, one adapts its links, while in the last model one adapts the methods of its neighbors.

\section{What is internal and external control?}

Some branches of systems science focus on internal control, while others aim for external control. In the engineering approach, there is an agent (the engineer) outside the system, who puts a goal on the system and who manipulates the system so that it would reach the goal. This agent thus aims for external control - control over the environment. With second-order cybernetics came the autonomous approach: the goal came inside the agent, who acts in the environment in order to reach its goal. Subjectivity came into the picture, where systems could self-organize and pursuit their own goals. Here, internal control became more important (Heylighen \& Joslyn, 2001).

The general scheme in cybernetics is a system that gets certain inputs (perception), which he transforms into an output (action) in order to reach goal(s). This output than changes the input the system receives from the environment. Note however that this is completely symmetric: system and environment could simply be switched, both are transforming inputs into outputs. But in general it is assumed there is some asymmetry: one (the system) is more powerful than the other (the environment) (Heylighen \& Joslyn, 2001). But what does power mean in this context? Inputs and outputs are affected in a mutual way, and it is a priori difficult to say whether an output is changed because it is manipulated by an input, or because it wants to change this input.

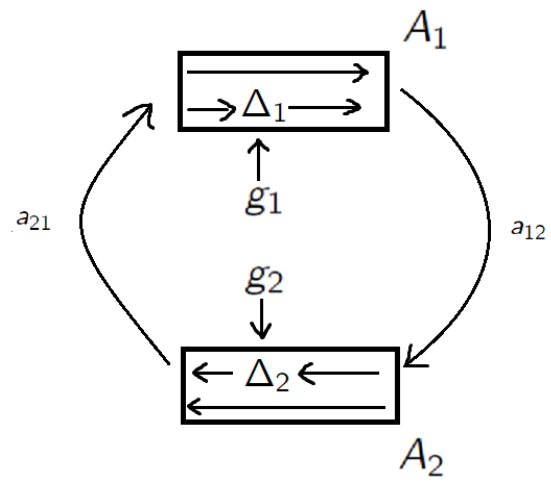

Figure 1: Coupled system

To answer this question, we consider such a coupled system (see figure 1). We have two agents, $A_{1}$ and $A_{2}$, both with goal(s) $g_{1}$ and $g_{2}$. $A_{1}$ sends a signal $a_{12}$ to $A_{2}$, which sends back a signal $a_{21}$. $A_{1}$ transforms this input $a_{21}$ into a $\Delta_{1}$, which he then uses to create the output. If $g_{1}$ is just one value, $\Delta_{1}$ can be seen as the difference between the real value and the goal state, but in general $\Delta_{1}$ can be seen as the part that matters. This means: that part of the variables of the input that $A_{1}$ cares about, in the 
composition he cares about. For example, he might want to have one variable bigger than another, but doesn't care how much the difference is. $\Delta_{2}$ plays the same role for $A_{2}$.

An agent would feel in power if the actions he performs can affect what he cares about, this is $\Delta_{1}$. This can be seen as a definition of internal control (IC):

$$
I C\left(A_{1}\right)=\left|\frac{d \Delta_{1}}{d a_{12}}\right|(1)
$$

By this, I mean the difference in result given a standard difference in action. Off course, this depends on the kind of action an agent performs: it might be that changing one variable doesn't have any effect, while changing another does, or that only after a certain threshold an action has effect. An agent will feel powerless if any action he does, gives the same result, thus if he doesn't have internal control.

On the other hand, an agent has power over another agent if it can influence the state of the other agent, as in the state that matters for the agent. We then say that the agent has external control, which we thus define as:

$$
E C\left(A_{1}\right)=\left|\frac{d \Delta_{2}}{d a_{12}}\right|
$$

Again, this depends on what kind of actions are performed, and $\Delta_{2}$ can also be affected in different ways. There is an assumption here that we know what is important for $A_{2}$, that $\Delta_{2}$ is known.

However, we can also look at everything from the perspective of what happens to $A_{1}$. We thus look at the external control exercised on $A_{1}$, this is $E C\left(A_{2}\right)=\left|\frac{d \Delta_{1}}{d a_{21}}\right|$, and the internal control of $A_{1}(1) . A_{2}$ can here be seen just as an environment, with some unknown complex dynamics, and without the assumption that we know what is important for $A_{2}$ (or even that there is a $\Delta_{2}$ ). We can than look what a standard difference in action from $A_{1}$ does. This $d a_{12}$ will give rise to a difference in the input of $A_{1}$, this is $d a_{21}$. This input will change the $\Delta_{1}$ of $A_{1}$ in an amount given by $d \Delta_{1}$. This $d \Delta_{1}$ is the same in $I C\left(A_{1}\right)$ and $E C\left(A_{2}\right)$. Thus if $d a_{21}>d a_{12}, E C\left(A_{2}\right)<I C\left(A_{1}\right)$. In words, if a small difference in action of $A_{1}$ can lead to big changes in the input it receives, then that agent has more internal control than the external control exerted on it. On the other hand, if $d a_{21}<d a_{12}$, then $E C\left(A_{2}\right)>I C\left(A_{1}\right)$. Thus, if one's actions doesn't lead to a lot of results, he'll have less internal control than the external control put on him.

Internal and external control are not necessary related, as following example shows (see figure 2). Consider a coupled system, where agent $A_{1}$ tries to control variable $a$, while $A_{2}$ wants to control $b$. $A_{1}$ puts the variables at state $\left(a_{12}, b_{12}\right)$ and sends this to $A_{2}$, who vice versa transforms it into $\left(a_{21}, b_{21}\right)$. As a classical cybernetic system, each agent moves the variable it is controlling closer to its goal state, for $A_{1}$ this means $a_{12}=a_{21}+k_{1} \Delta_{1}$, with $\Delta_{1}=g_{1}-a_{21}$ and $k_{1}>0$ a certain constant, and analogous for $A_{2}$. But what do the agents do with the variable that doesn't matter for them?

First consider the case where they put this variable at a certain constant, thus $b_{12}=c_{1}$ and $a_{21}=c_{2}$. Then, both agents have no internal control at all: no matter the action they do, the difference from the goal state will remain constant, for $A_{1}$ this is $\Delta_{1}=g_{1}-c_{2}$. They do however have complete external control: they can completely determine the $\Delta$ of the other, by choosing the constant they send. Thus $E C\left(A_{1}\right)=\left|\frac{d \Delta_{2}}{d b_{12}}\right|=\left|\frac{d\left(g_{2}-c_{1}\right)}{d c_{1}}\right|=1$ (considering a change in action of the second variable). Note that this way of acting could even be with the best intentions, to put the variable to a state the other agent prefers. But since an agent has imperfect knowledge of the goal(s) of another agent, he won't succeed completely, and even if he does, the other agent will still feel like he's not in control. 
Now consider on the other extreme the case where the agents don't affect the variable they don't care about. Thus $b_{12}=b_{21}$, and $a_{21}=a_{12}$. Here, agents have complete internal control: a change in action will affect their $\Delta . I C\left(A_{1}\right)=\left|\frac{d \Delta_{1}}{d a_{12}}\right|=\left|\frac{d\left(g_{1}-a_{12}\right)}{d a_{12}}\right|=1$. They however don't have any external control: they don't have any effect on the $\Delta$ of the other. Actually, we can see this as a completely decoupled system, where two agents simply try to control another variable, independent from each other.
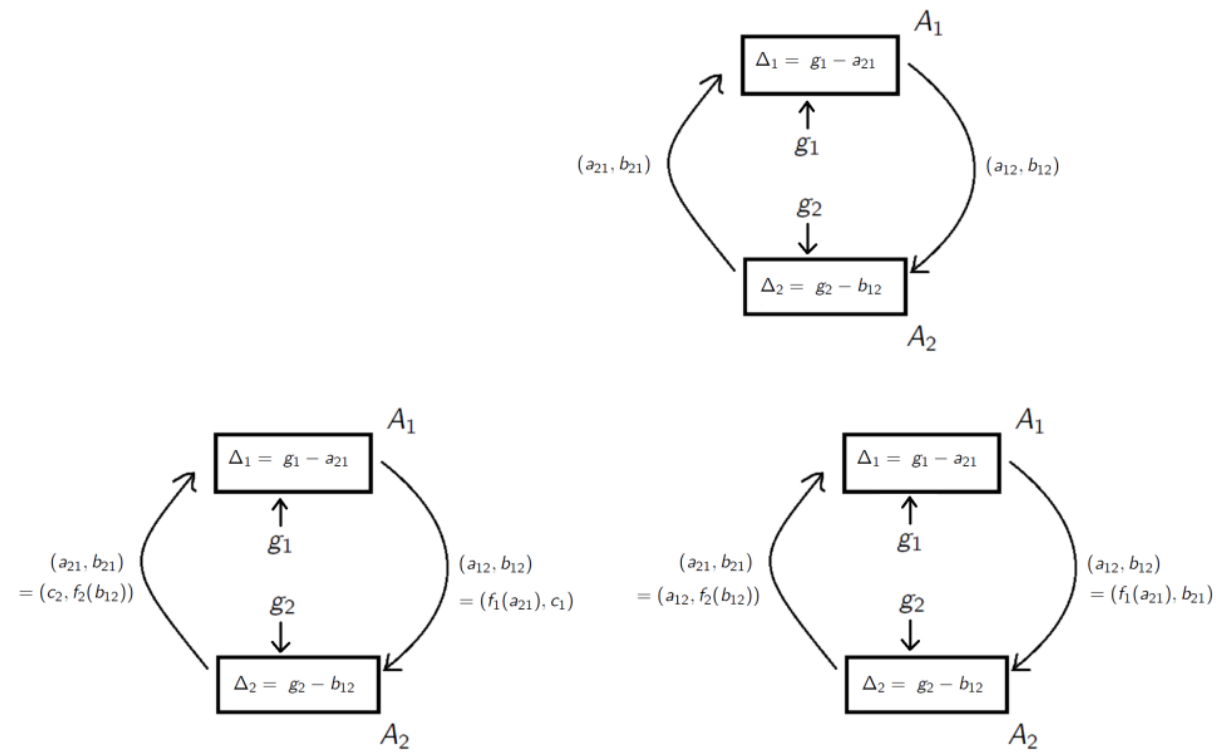

Figure 2: An example to show how internal and external control is not always related. Both agents control a different variable (upper). In the first case (lower left), they put the other variable to a constant, which results in external, but no internal control. In the second case (lower right), they don't affect the other variable, which result in internal, but no external control.

\section{Different strategies for control}

This clarified what I mean with internal and external control. Now, I'd like to use a multi-agent model to show in which ways agents can have internal or external control.

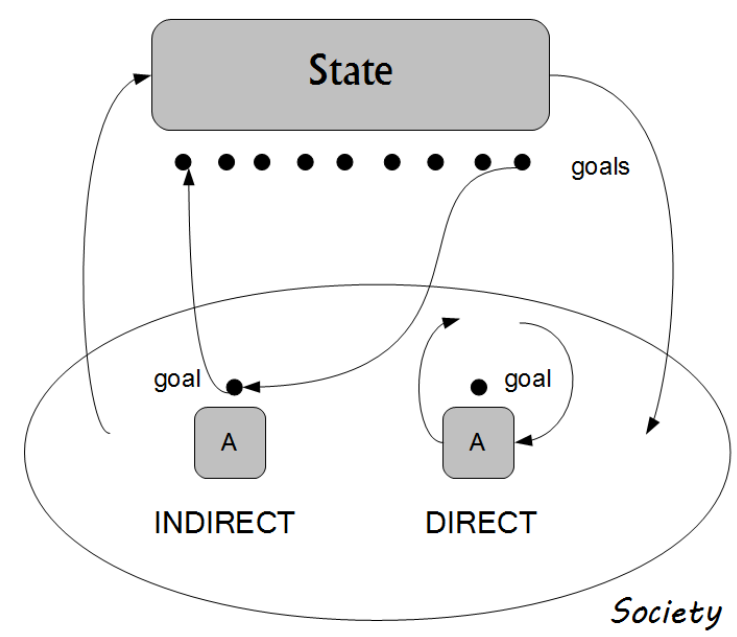

Figure 3: Direct versus indirect acts. $A$ is an agent, living in a society. The agent on the left tries to reach its goals indirectly. It will try to influence the goal(s) of the state. This state tries to reach its goals by acting in the society. The problem with this approach is that some goals of the state will also influence the goal of the agent. And since the state is a much bigger structure than an individual agent, the change in the agent will be much more significant. The agent on the right acts directly in an attempt to reach its goals, by acting in the society $A$ 
On the one hand, the difference between these strategies lies in the locality: one could develop local actions, or one could attempt to influence the global system.

Traditional politics still assumes people should get into power to impose their societal vision. People try to get global control to fulfill their needs. Prefigurative politics means someone already tries to put hir societal vision into practice today, i.e. goals and methods get aligned. Thus, aiming for a world where no-one is controlled, shouldn't happen by trying to control others. Anarchists ${ }^{1}$ using direct action apply this principle. Direct action means you directly act against a certain oppressive dynamic, in contrast to, for example, asking politicians to do something about it. An example is blocking an immigration detention center, so that they can't expel anyone that day.

With direct action, one tries to reach its goals directly. While working through the state means someone tries to influence the goals of the state, so that they include his goal, or so that his goals and the ones of the state are more mutual. Figure 3 shows this difference.

On the other hand, the difference lies in the way one acts, i.e. what one tries to change. An agent could adapt its links, or try to change either the methods or the goals of its neighbors. Adapting one's links means moving to a different environment. For example, someone can try to find friends who share ideas and like what he likes, or he can try to convince his friends to do what he wants to do. Another example looks to communication. Some people spread a message with the aim of convincing. They intend to change the goals of other people. While other people spread their ideas to create a dialogue: by getting inspiration and feedback, both parties can improve their thoughts. They change their connections in order to collaborate with the most interesting people, and improve the methods of their neighbors.

But these three ways are related, and it might be able to put them on a continuum. Changing one's links affect the possibilities her neighbors have, the methods they can use. And methods can be seen as putting a subgoal to reach a bigger goal. The question is then which goals are fundamental for an agent, and which are just means to an end. Probably this isn't that black-and-white, goals can be more or less important.

I will now give a general framework of a multi-agent model, which I will apply to existing models. These can be seen as toy models of the different strategies of control introduced above.

\section{Generalization}

The framework I develop here is a generalization of the model of Mesarovic (Mesarovic et al., 20oo). His model works with a hierarchical system where there is a 'top' and a 'bottom', and it differentiates between a process and a control system. I won't do this, I'll work with a general model of connected agents. An agent gets certain input, this can come from other agents in the form of coordination or feedback, or could come from 'outside', from the environment. It transforms this into an output, which can be a coordination input for other agents, or some general output going outside.

There is also a feedback signal created for every coordination input it received, which it sends back to the agent that send the coordination input. An agent uses his inputs and outputs to create a feedback signal.

Thus, an agent sends a certain signal, called coordination input, to influence its neighbors. It gets back a feedback signal as information of how well it succeeded in its attempts. We'll work with a directed network. The inputs and outputs of an agent $i$ are shown in figure 4 . A link between agent $i$ and agent $j$, means $i$ sends a coordination input $c_{i j} \in C_{i j}$ to $j$, where $c_{i j}$ is the specific coordination input, and $C_{i j}$ is the set of possible coordination inputs from $i$ to $j$. Consequently, $j$ sends a feedback signal $f_{i j} \in F_{j i}$ to $i$. Further on, it's possible an agent receives some input from its environment $\Omega_{i}$ and 
send some output $y_{i} \in Y_{i}$ outside. I'll consider these also as coordination inputs and outputs, thus I define the set of coordination inputs as $C_{i}^{-}=\Omega_{i} \times \prod_{j} C_{j i}$ and the coordination output as $C_{i}^{+}=Y_{i} \times \prod_{j} C_{i j 2} . F_{i}^{-}=\prod_{j} F_{j i}$ is the feedback an agent receives, while $F_{i}^{+}=\prod_{j} F_{i j}$ is the feedback an agent sends. Thus the functions to create a coordination and feedback signal are the following:

$$
\begin{gathered}
C_{i}: C_{i}^{-} \times F_{i}^{-} \rightarrow C_{i}^{+}(2) \\
F_{i}: C_{i}^{-} \times F_{i}^{-} \times C_{i}^{+} \rightarrow F_{i}^{+}(3)
\end{gathered}
$$

An agent uses its coordination and feedback inputs to create a coordination output (2), and takes all of this to generate a feedback signal (3).

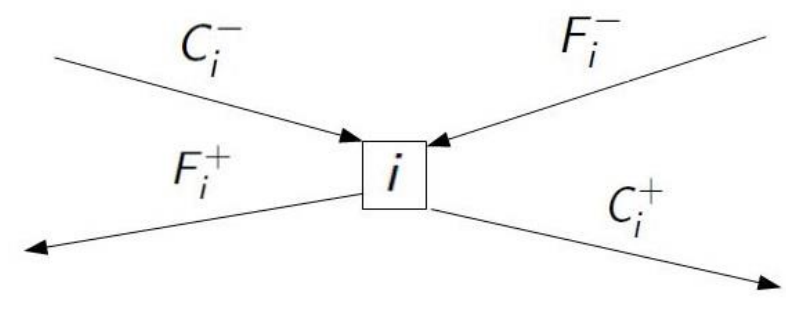

Figure 4: Inputs and outputs of an agent in the general model

\subsubsection{Coordinability}

The idea of coordinability is that we want that all agents find a solution to their decision problem. We'd further want this solution to be stable: we don't want that there is only a solution for an insignificant amount of time.

To introduce this decision problem, we decompose $C_{i}$ into two subsystems:

$$
\begin{aligned}
& d_{i}: C_{i}^{-} \times F_{i}^{-} \rightarrow X_{i}(4) \\
& c_{i}: F_{i}^{-} \times X_{i} \rightarrow C_{i}^{+}(5)
\end{aligned}
$$

so that $C_{i}=c_{i}$ 。 $d_{i}$, by first applying $d_{i}$ and then $c_{i}$, we get back the original function $C_{i}$ (2). The idea is that for every input $\left(c_{i}^{-}, f_{i}^{-}\right)$, there is a decision problem $D_{i}\left(c_{i}^{-}, f_{i}^{-}\right)$. The solution(s) to this problem are defined by $\left.d_{i}\left(c_{i}^{-}, f_{i}^{-}\right)(4)\right)$. This is thus a set of possible solutions, it could be empty or contain multiple solutions. This solution is then implemented to create a coordination output, given the feedback it received (by (5)). We define following predicate to say that something is a solution to a decision problem:

$$
P(x, D) \equiv x \text { is a solution of } D
$$

First consider the case where there is no feedback. Then we define stable internal coordinability as:

$$
\exists \bar{x}: \forall i P\left(x_{i}, D_{i}\left(c_{i}^{-}\right)\right), \text {with } c_{i}^{+}=c_{i}\left(x_{i}\right)(6)
$$

where $\bar{x}=\left(x_{1}, \ldots, x_{n}\right)^{3}$. This means that there exists a solution to all decision problems given a certain input (the part $P\left(x_{i}, D_{i}\left(c_{i}^{-}\right)\right)$), and this input is constructed given these solutions (the part $c_{i}^{+}=c_{i}\left(x_{i}\right)$ ). This last part makes that we have a stable solution, if we omit the condition $C_{i}^{+}=c_{i}\left(x_{i}\right)$, we get a weak version of internal coordinability.

If each decision problem has one solution (thus $d_{i}$ is a function), condition (6) is equal to: 


$$
\begin{gathered}
\exists \bar{c}: \forall i c_{i}^{+}=C_{i}\left(c_{i}^{-}\right)(7) \\
\text { where } \bar{c} \in \prod_{i, j} C_{i j} \times \prod_{i} \Omega_{i} \times \prod_{i} Y_{i} .
\end{gathered}
$$

Now, with feedback, stable internal coordinability is defined as follows:

$$
\exists \bar{x}, \bar{f}: \forall i P\left(x_{i}, D_{i}\left(c_{i}^{-}, f_{i}^{-}\right)\right) \wedge f_{i}^{+}=F_{i}\left(c_{i}^{-}, f_{i}^{-}, c_{i}^{+}\right) \text {, with } c_{i}^{+}=c_{i}\left(f_{i}^{-}, x_{i}\right) \text {. }
$$

where $\bar{f} \in \prod_{i, j} F_{i j^{4}}$. This thus means that there is a solution and feedback signal, such that the feedback signal remains stable (the part $f_{i}^{+}=F_{i}\left(c_{i}^{-}, f_{i}^{-}, c_{i}^{+}\right)$), and that the coordination input that gets used, is constructed from the solution (thus that there is stability for the coordination inputs, this is the part $\left.c_{i}^{+}=c_{i}\left(f_{i}^{-}, x_{i}\right)\right)$.

If the $d_{i}$ s are functions, (8) is equivalent to:

$$
\exists \bar{c}, \bar{f}: \forall i c_{i}^{+}=C_{i}\left(c_{i}^{-}, f_{i}^{-}\right) ; f_{i}^{+}=F_{i}\left(c_{i}^{-}, f_{i}^{-}, c_{i}^{+}\right)(9)
$$

Now, it is also possible that we want that a certain given problem $D$ has a solution. We can assume the solutions to this problem are in $Y=\bigcup_{i} Y_{i}$. There is a function $\pi: X \rightarrow Y$ (part of the $c_{i}$ 's will do this). We can then define stable coordinability relative to a problem $D$ by adding the condition $P(\pi(\bar{x}), D)$.

\subsection{Application to the controllability of complex networks}

I would now like to apply this framework to the theory of the controllability of complex networks (Liu, Slotine, \& Barabasi, 2011). The idea here is that you try to control a network by sending certain inputs to certain nodes. This is thus a model of global control by adapting the goals of agents. Liu et al. searched for a minimal set of nodes which one has to control to have control over the whole network. In this model, each node $j$ has a value $X_{j}$ which got influenced by the values of their neighbors and the control input. This happens by the following update:

$$
X_{j} \leftarrow X_{j}+\sum_{i} a_{j i} X_{i}+\sum_{k} b_{j k} u_{k}
$$

where $a_{j i}$ is the link weight between $X_{i}$ and $X_{j}$, and $b_{j k}$ is the link weight from the controller $u_{k}$ to $X_{j}$ ${ }^{5}$. Liu et al. argue that the exact values of $a_{j i}$ and $b_{j k}$ don't matter for the controllability. We can write this into our framework by taking $X_{j}$, the output of $j$, also as input of $j$. We take $a_{i i}=1 \forall i$. An agent sends the same output to all agents. We got:

$$
\begin{aligned}
C_{i}: C_{i}^{-} & \times \Omega_{i} \rightarrow C_{i}^{+} \\
X_{i} \leftarrow \sum_{j} a_{i j} X_{j}+\sum_{k} b_{i k} u_{k} & =C_{i}(X, U)
\end{aligned}
$$

where $X_{j} \in C_{i}^{-}, u_{k} \in \Omega_{i}, X=\prod_{j} X_{j}$ and $U=\prod_{j} u_{j}$. Thus $X_{i}$ is a function of $X$ and $U$.

Liu et al. define controllability as being able to put the network in any desired state. It isn't necessary however that this is a steady state. We can try to steer to this state by choosing certain inputs. We call this version weak controllability, and it can be defined as a form of weak coordinability, namely when there is weak coordinability relative to all decision problems, thus

$$
\forall D \exists \bar{x}: \forall i P\left(x_{i}, D_{i}\left(C_{i}^{-}\right)\right) \wedge P(\bar{x}, D)
$$

Notice that the implementer is here the identity function; the solution $x_{i}$ of the decision problem gets send out. $d_{i}$ is a function (equal to $C_{i}$ ), thus the first part is actually always true, though we 
would like the $C_{i}^{-}$to come from a previous step. The second part states that we should find an $\bar{x}$ that is a solution to the decision problem, for all the decision problems. A decision problem is a subset of possible $\vec{x}$ s, thus this is equivalent of stating it's true for all $\vec{x}$ s.

The theory of controllability found out that you have controllability if each node has its own direct superior, this can be another node or an input node. This leads them to the theorem that a minimal set of nodes you have to control is equal to the unmatched nodes in a maximum matching.

The requirement that you should reach a stable state could however be useful. Reaching a desired state for only a millisecond, is often not what you want. I thus define stable controllability as:

$$
\forall \bar{c} \exists \bar{u}: \forall i c_{i}^{+}=C_{i}\left(c_{i}^{-}, \bar{u}\right) \cdot(11)
$$

We can again define this by seeing it as an overall decision problem. The solutions of the decision problem are in $\bar{C}$. It is a solution of the problem if it is equal to our predefined desired state. Thus, controllability means the following is true for all decision problems:

$$
\exists \bar{c}, \bar{u}: \forall i c_{i}^{+}=C_{i}\left(c_{i}^{-}, \bar{u}\right) \wedge P(\bar{c}, D) .
$$

Internal stable coordinability (from (7)) is here defined as:

$$
\exists \bar{c}, \bar{u}: \forall i c_{i}^{+}=C_{i}\left(c_{i}^{-}, \bar{u}\right)(12)
$$

which is thus less strong - there only needs to be one stable solution.

Hence coordinability looks whether the agents can coordinate between each other, while controllability wants them to behave in a specific externally defined way.

I'd now want to check whether there is stable controllability (11) in this model. Thus, we consider $X_{i}$ fixed for all $i$. We find that we want to find $u_{k}$ 's such that (10) is an equality, thus

$$
\sum_{i} a_{j i} X_{i}+\sum_{k} b_{j k} u_{k}=0 \forall j
$$

where we don't consider $a_{j j}$, thus we put this at 0 again.

A little bit of calculation, learns that if for a certain $j, b_{j k}=0 \forall k$, then we should have $S_{j}:=-\sum_{i} a_{j i} X_{i}=0$. Otherwise, we should define one $u_{l}$ as depending on the others by the formula:

$$
u_{l}=\frac{S_{j}-\sum_{k \neq l} b_{j k} u_{k}}{b_{j l}}
$$

Thus, each node for which $S_{j} \neq 0$ should have its own control input, which means almost all nodes should be controlled.

If we take $X_{i}=0 \forall i$, we find a solution for stable internal coordinability (from (12)).

This approach fits in the engineering approach of first-order cybernetics, where the goal is appointed to the system from the outside. The aim here is thus external control. The assumption is that there is a given and completely known complex network, and one wants to control its dynamics.

\subsection{Self-organized control}

I would now like to extend the above model to allow feedback. The idea is that we see the feedback as link weight. The link weight is changed so that the input an agent receives fulfills its desire more. The link weight $w_{i j}$ gives the strength of the connection between $i$ and $j$, this is changed depending on how useful the value of $j$ is. I thus consider it as the feedback $F_{j i}$ that $j$ sends to $i$. This is thus a model where an agent changes his environment in order to get control. The agents in this model 
hence have goals on their own, in contrast with the previous model. I model this by giving each agent a reference value $R_{i}$. An agent wants to move its value $X_{i}$ to the reference value. The updating of a value of an agent happens as above, except that I don't allow any external input anymore (13). The coordination an agent sends is its value $X_{i}$ multiplied with the link weight (14) (this operation thus happens with the sending agent instead of with the receiving agent). I consider two loops: an agent sends its updated value and the constructed feedbacks also to himself, so that its first output, and then gets input. I thus get:

$$
\begin{gathered}
C_{i}: \underbrace{X_{i}}_{\in C_{i}^{+}} \leftarrow \underbrace{X_{i}}_{\in C_{i}^{-}}+\sum_{j} \underbrace{C_{j i}}_{\in C_{i}^{-}(13)} \\
\underbrace{C_{i j}}_{\in C_{i}^{+}} \leftarrow \underbrace{F_{j i}}_{\in F_{i}^{-}} \underbrace{X_{i}}_{C_{i}^{-}(14)} \\
F_{i}: \underbrace{F_{i j}}_{\in F_{i}^{+}} \leftarrow F_{i j}+\alpha\left(R_{i}-X_{i}\right) X_{j} \\
=\underbrace{F_{i j}}_{\in F_{i}^{-}}+\alpha(R_{i}-\underbrace{X_{i}}_{\in C_{i}^{+}} \underbrace{C_{j i}}_{\in C_{i}^{-}} / \underbrace{F_{i j}}_{\in F_{i}^{-}}
\end{gathered}
$$

The last formula (15) for the updating of the link weight comes from the theory of perceptron learning (Haykin, 1994). If the total input is too big $\left(X_{i}>R_{i}\right)$, the link weight (feedback) is weakened for positive inputs, and strengthened for negative ones, so that the total input becomes less. The opposite happens if the total input is too little. Figure 5 shows how these functions work.

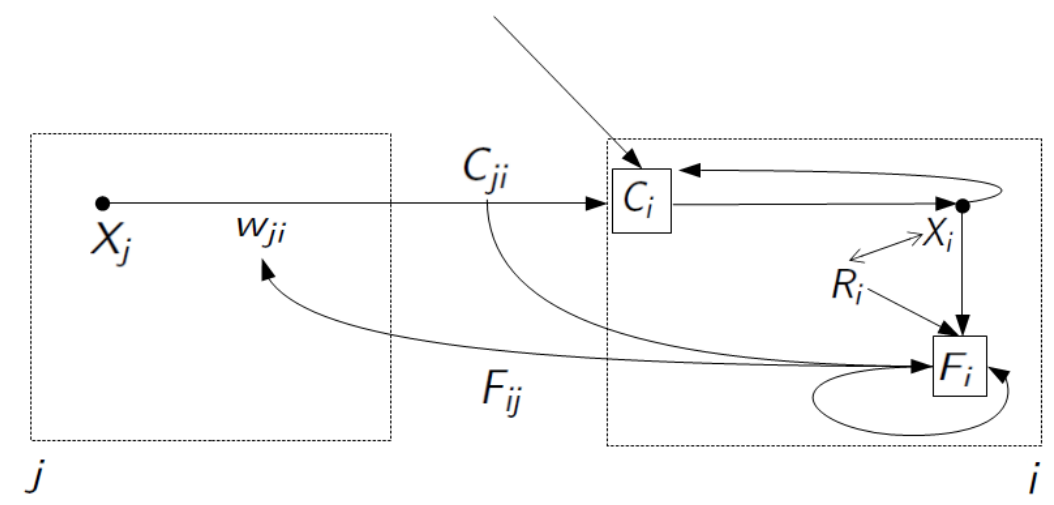

Figure 5: A graphical representation of the formula's (13), (14) and (15) of self-organized control. Agent $j$ combines its value $X_{j}$ and the link weight $w_{j i}$ to generate a coordination signal $C_{j i}$. This input is used by agent $i$ in the processes $C_{i}$ and $F_{i}$. In $C_{i}$, the inputs agent $i$ receives is combined with its value $X_{i}$ to update this $X_{i}$. In $F_{i}$, this $X_{i}$ is then compared with $R_{i}$ to update the feedback signal $F_{i j}$ . This feedback changes the link weight $w_{j i}$ between $j$ and $i$.

I'd now like to know whether there is stable internal coordinability in this model. This is the case if there is a solution for the above equations (from (9)). Some calculation learns that there are two possible solutions. One is $X_{j}=0 \forall j$, then the feedback can be chosen at random. Another possibility is to take $X_{i}=R_{i} \forall i$. If $R_{j}=0 \forall j$, any feedback will satisfy. If not, there is a $k: R_{k} \neq 0$. For $j \neq k$, we can then take $F_{i j}$ at random, and then take

$$
F_{i k}=\frac{-\sum_{j \neq k} F_{i j} R_{j}}{R_{k}}
$$


for every $i$.

This last solution seems most logical, namely that the agents want to have their values equal to their reference values. We can put this as an overall decision problem: $R_{i}=X_{i} \forall i$. Then we get that the above solution is the only possible case of stable coordinability relative to this overall decision problem.

Thus, we find that there is stable coordinability in this model, if we assume the feedback (link weight) isn't bound to only positive numbers or only between 0 and 1 . However, the fact that there is a solution, doesn't necessary mean that this solution will be reached by this process. It might never get into this attractor. For example, if we take $X_{i}=0 \forall i$, none if the $X_{i}$ 's will change, thus it won't be able to reach a reference value if this value is different than zero. Also, if the learning parameter $\alpha$ is too high, agents might constantly overcompensate, thus never reach the reference value.

Thus, also in a weak version, coordinability won't always be reached. In which circumstances, it is and isn't, remains an open question, though it is plausible that often it is reached, since the model is built so as to go to the solutions (the update of the feedback signal makes it closer to its reference value).

This model is more in line with the autonomous approach of second-order cybernetics. Each agent has its own goal, which it tries to reach by adapting its links with other agents. In a social system, these links can be friendship ties, where on the internet it can represent how strong two people connect. For example, if you put an unwanted email in your spam folder and indicate that you don't want to receive any of these messages anymore, your weakening your link with the sender. In this way, you give some feedback on how much an email is wanted. Connecting with people with whom you share interests and detach from people who block you in reaching your goals, is another example.

\subsection{Control by changing the method of your neighbors}

I now want to construct a model where agents try to influence the methods of their neighbors. I will base this on Perceptual Control Theory (McClelland, 2004).

In perceptual control theory, an agent tries to control its perception $X_{i}$, by trying to equalize it with a certain reference value $R_{i}(16)$. But there are also other agents who try to control the same perception, with other reference values in mind, and the perception might also get disturbed by the environment $\Omega_{i}(17)$. Usually these disturbances are random, thus this isn't much of a problem. The model thus looks like this:

$$
\begin{gathered}
C_{i} \leftarrow C_{i}+\alpha\left(R_{i}-X_{i}\right)(16) \\
X_{i} \leftarrow \sum_{j} C_{j}+\Omega_{i}
\end{gathered}
$$

In this model, the perception will converge to an average of the reference values, where $\alpha$ represents the power an agent has. This can thus be a simple model of social power. If all the other agents have the same reference value, the one (or minority of) agent(s) with another reference value won't be able to match its reference value. In general, this one control loop is part of a perceptual hierarchy, with different reference values which are controlled by a higher control loop. If there is conflict because a reference value is constantly not met, it will perturb this reference value until it is met. Thus, an agent will conform to the pressure of the group of all the same reference values. 
Inspired by this model, I now construct a model where the idea is that one tries to influence your neighbors to send the right coordination input, by sending them certain feedback. We assume the coordination an agent $j$ sends to $i$ is constructed as follows:

$$
C_{j i} \leftarrow C_{j i}+F_{i j(18)}
$$

Each agent $i$ has a value $X_{i}$ he wants to put as close as possible to its reference value $R_{i .} X_{i}$ is constructed as follows:

$$
X_{i} \leftarrow \sum_{j} C_{j i}(19)
$$

The way an agent tries to control its neighbors to send coordination which satisfies its needs (reference value) more, is by sending this feedback:

$$
F_{i j} \leftarrow \alpha\left(R_{i}-X_{i}\right)(20)
$$

A shortcoming of this model is that the coordination input is assumed to be known and of a specific form, so that it satisfies our urge to control it. That's why I want to generalize the model where we assume the coordination function is unknown, and we don't even know how exactly this gets aggregated into $X_{i}$. Thus we just assume

$$
X_{i} \leftarrow f\left(C_{i}^{-}\right)
$$

with $f$ some unknown function. Then we can still try to get control by looking how our $X_{i}$ got affected by the $F_{i}^{+}$we have sent out (we send the same feedback signal to all agents, thus we assume $F_{i}^{+}=F_{i j} \forall j$ ). If there is a positive correlation, thus a bigger $F_{i}^{+}$results in a bigger $X_{i}$, then we can use the same update mechanism as above. If there is a negative correlation however, we should do the opposite, subtracting instead of adding. We thus get the following formula's:

$$
\begin{array}{lll}
F_{i}^{+} \leftarrow F_{i}^{+}+\alpha\left(R_{i}-X_{i}\right) & \text { if } & F_{i}^{+} \nearrow \Rightarrow X_{i} \nearrow \\
F_{i}^{+} \leftarrow F_{i}^{+}-\alpha\left(R_{i}-X_{i}\right) & \text { if } & F_{i}^{+} \nearrow \Rightarrow X_{i} \searrow
\end{array}
$$

This still has some unrealistic assumptions though, because the aims and the methods got separated. The $X_{i}$ and the output $C_{i j}$ send out are completely separated. We see what kind of consequences this has when we check whether there is stable internal coordinability (9). It's difficult to check this for the general model, so I do this for the more specific model (thus (18),(19) and (20) should be equations). There is stable internal coordinability if

$$
\begin{array}{r}
0=F_{i j}=\alpha\left(R_{i}-X_{i}\right) \\
\Rightarrow R_{i}=X_{i}
\end{array}
$$

And

$$
R_{i}=X_{i}=\sum_{j} C_{j i}
$$

One can easily choose $C_{j i}$ 's such that this is fulfilled.

This model is again in line with the autonomous approach, where all agents have a goal they try to reach. Here however, they do so by controlling the methods of other agents. In a social context, you can see this as someone who want others to say what he wants to hear, and do as he wants. As long as these things are independent from the goals of the person asked, this may well work. But because of this decoupling, the result is often artificial, where people are just saying what one wants to hear, but without being really committed to it. 


\section{Conclusion}

I first examined what internal and external control means in cybernetic terms. I found out that these are two distinct things, and having more of the one doesn't necessary increase the other, sometimes the opposite is even true.

I also looked into different models of control in this paper. We can put this in the framework of the scope of influence and the way one acts, as discussed in section 3. The scope of influence tells how local or global one acts, while the way one acts tells whether one tries to influence the links, methods or goals of neighbors.

The first group of models try to control a whole network, they work globally. The model of controllability is an example of this, where one tries to influence the goals of the agents. Another example is the model of Emergent Control (Kreyssig \& Dittrich, 2011), where they try to achieve a global goal by adapting the local rules (methods). We saw that at least in the model of controllability, this is difficult to achieve because one has to control almost all the nodes, pushing it away from its natural state.

Other models work locally, they assume the agents want to get control. In the model of selforganized control, they did this by changing the links they had with other neighbors. This worked, under the assumption that the feedback (link weights) wasn't bound too much. In the second model of this kind, agents tried to adapt the methods of their neighbors. This also worked, but there was the implicit assumption that goals and methods are separated, which isn't very realistic.

A general shortcoming of these models is that we assume the goal of an agent is simply to reach a certain reference value, while in reality goals are usually far more implicit and multidimensional. It might even be better to speak about certain value systems instead of certain goals, where there isn't one optimal solution. But this is more difficult to formalize, and the general principles presented in this paper seem to be also true in this case. I.e. that it's easier to get control over one's life by acting locally, as least as possible disturbing the core values of your environment. It might even be more easy to do so in reality, because there are far more possibilities to satisfy one's values.

\section{References}

Gelderloos, P. (2007). Insurrection vs. organization. The Anarchist Library.

Haykin, S. (1994). Neural Networks: A Comprehensive Foundation (1st ed.).Upper Saddle River, NJ, USA: Prentice Hall PTR.

Heylighen, F., \& Joslyn, C. (2001). Cybernetics and Second-Order Cybernetics. In Encyclopedia of Physical Science E Technology (3 rd. Academic Press.

Kreyssig, P., \& Dittrich, P. (2011, January). Emergent control. In C. Mller-Schloer, H. Schmeck, \& T. Ungerer (Eds.). Organic computing a paradigm shift for complex systems (pp. 67 78 ). Springer Basel. Retrieved 2014-10-27, from http://link.springer.com/chapter/10.1007/978-3-0348-0130-o 4

Liu, Y.-Y., Slotine, J.-J., \& Barabasi, A.-L. (2011, May). Controllability of complex networks.

Nature, 473 (7346), 167\{173. Retrieved 2014-10-16, from

http://www.nature.com/nature/journal/v473/n7346/abs/nature10o11.html doi: 10.1038/nature1oo11 
McClelland, K. (1994, December). Perceptual control and social power. Sociological Perspectives, 37 (4), 461\{496. Retrieved 2014-10-16, from http://spx.sagepub.com/content/37/4/461 doi: 10.2307/1389276

McClelland, K. (2004, January). The collective control of perceptions: constructing order from conflict. International Journal of Human-Computer Studies, 60 (1), $65\{99$. Retrieved 2014-02-12, from http://www.sciencedirect.com/science/article/pii/S1071581903001381 doi: 10.1016/j.ijhcs.2003.08.003

Mesarovic, M. D., Macko, D., \& Takahara, Y. (200o). Theory of hierarchical multilevel systems. Retrieved 2014-02-12, from http://cds.cern.ch/record/1611599

Salthe, S. N. (2013). Evolving hierarchical systems: their structure and representation. Columbia University Press.

Stewart, J. E. (2014). The direction of evolution: The rise of co-operative organization. Biosystems. Retrieved 2014-06-10, from http://www.sciencedirect.com/science/article/pii/S03032647140008oX. doi: 10.1016/j.biosystems.2014.05.006

Taleb, N. N. (2012). Antifragile: Things that gain from disorder. Penguin UK.

\footnotetext{
${ }^{1}$ An anarchist is someone who wants to organize society without hierarchy or domination. See for example Gelderloos, P. (2010). Anarchy Works for examples and methods of how people organize in an anti-authoritative way. $2 \times$ and $\Pi$ denotes the Cartesian product of sets. It is used to be able to put all the different coordination inputs (or outputs) in one variable. For example, a specific coordination output $c_{i}^{+} \in C_{i}^{\dagger}$ can be seen as a vector $\left(\mathrm{y}_{1}, \mathrm{c}_{\mathrm{i} 1}, \ldots, \mathrm{c}_{\mathrm{in}}\right)$. The - denotes that it's input for $i$, while + denotes that its output.

${ }^{3} \exists_{i}$ means 'there exists and $i$ (for which ....) $\forall_{i}$ means 'for all $i$ (applies ....)'. Thus, in words, stable internal coordinability means: there exists an $X$, so that for all $i$ it is true that $X i$ is a solution of $D_{i}\left(c_{i}{ }^{-}\right)$, with $c_{i}{ }^{\dagger}{ }^{=} c_{i}\left(X_{i}\right)$.

${ }^{4} \wedge$ denotes the 'and' symbol, thus both conditions should be true. $F_{i}$ is the function defined in (3).

${ }^{5}$ The symbol $\leftarrow$ means that at each time step, the value $X_{j}$ gets updated into the formula on the right. Thus, at each time step, the weighted sum of the value of its neighbors and controllers is added to the value of a node.
} 